\title{
The present status of the EPS nuclear PDFs
}

\author{
Hannu Paukkunen ${ }^{* a}$, Carlos A. Salgado ${ }^{a}$ and Kari J. Eskola ${ }^{b}$ \\ ${ }^{a}$ University of Santiago de Compostela \\ Departamento de Física de Partículas and IGFAE, Universidade de Santiago de Compostela, \\ Spain \\ ${ }^{b}$ University of Jyväskylä \\ P.O. Box 35, FI-40014 University of Jyväskylä, Finland \\ Email: hannu.paukkunen@usc.es, carlos.salgado@usc.es, \\ kari.eskola@phys.jyu.fi
}

The recent global analyses of the nuclear parton distribution functions (nPDFs) lend support to the validity of the factorization theorem of QCD in high-energy processes involving bound nucleons. With a special attention on our latest global analysis EPS09, we review the recent developements in the domain of nuclear PDFs.

35th International Conference of High Energy Physics

July 22-28, 2010

Paris, France

${ }^{*}$ Speaker. 


\section{Introduction}

The global analyses of nPDFs approach the experimentally observed differences between the hard-process cross-sections involving bound and free nucleons, $\sigma^{\text {boundnucleon }} \neq \sigma^{\text {freenucleon }}$, with the toolkit of perturbative QCD (pQCD). Different processes in different kinematical regions are studied in order to extract the universal - process independent - nuclear PDFs, and to estimate the conditions in which the factorization theorem of QCD appears applicable. In this talk we recapitulate the essentials of the latest offspring of the EPS-generation [1, 2, 3, 4], EPS09 [5], and release a piece of news about the recent EPS-activities.

\section{Analysis}

\subsection{Experimental Input}

The main source of experimental input in EPS09 comes from the measurements of deep inelastic scattering (DIS) and Drell-Yan (DY) dilepton production. These data alone, however, leave the gluon PDFs badly unconstrained. As noted in [5], part of such freedom can be reduced in a way that do not cause disagreement with other data - by adding data for average high- $p_{T}$ pion production in $\mathrm{d}+\mathrm{Au}$ collisions measured at RHIC. It should be noted that the fragmentation functions which in $\mathrm{pQCD}$-calculations turn the partons to pions might also experience a modification with respect to the free proton fragmentation functions [6]. This effect is not accounted for in EPS09 where the pion-observables are mainly needed for constraining the nuclear gluons.

\subsection{Theoretical Framework}

In EPS09, the bound proton PDFs for each flavor $i$ are linked to the free proton ones by

$$
f_{i}^{\text {bound proton }}\left(x, Q^{2}\right) \equiv R_{i}^{A}\left(x, Q^{2}\right) f_{i}^{\text {freeproton }}\left(x, Q^{2}\right) .
$$

The nuclear modification factors $R_{i}^{A}$ are parametrized at charm quark mass treshold $Q^{2}=Q_{0}^{2}=$ $1.69 \mathrm{GeV}^{2}$, assuming a flavor-independent nuclear modification for light sea quarks $R_{\bar{u}}^{A}=R_{\bar{d}}^{A}=R_{\bar{s}}^{A}$, and valence quarks $R_{u_{V}}^{A}=R_{d_{V}}^{A}$ at $Q_{0}^{2}$. This is, of course, just a simplifying assumption and our plan is to to release this assumption in a future.

The EPS09 employ the $\overline{M S}$, zero-mass variable flavor number scheme. Accordingly, the baseline free proton PDF set used was the CTEQ6.1M [7], which probably remains as the last CTEQset in this simple scheme, the more recent ones involving a more complete prescription to treat the heavy quarks. Recently, we have also implemented such a scheme to our fitting engine. However, the available nuclear data are not very sensitive to the heavy quarks, and our tentative results do not display large deviations from the zero-mass ones. Therefore, the usage of EPS09 with newer sets of the free proton PDFs like CT10 [8] should be justified.

\subsection{Fit Quality}

In order to find the $R_{i}^{A}$ s that optimally reproduce the experimental data, a certain quality criterion is need. We minimize a generalized $\chi^{2}$-function which is essentially

$$
\chi^{2} \equiv \sum_{N} w_{N} \sum_{i \in N}\left(\frac{D_{i}-T_{i}}{\sigma_{i}}\right)^{2},
$$


where $D_{i}$ and $\sigma_{i}$ are the experimental data points with their errors, and $T_{i}$ is the corresponding calculated value. A small extension to this due to the additional normalization uncertainty of the pion data is explained in [5]. The additional weights $w_{N}$ are assigned to emphasize certain data sets with only a small amount of data points but carrying physically relevant information. Partly, this is a technical issue aiming to improve the convergence of the fit with more open parameters, but certainly introduce some subjectivity to the central fit. Unfortunately, the weights also distort the error analysis explained shortly, and inevitably lead to a somewhat underestimated PDF-errors. Reducing the impact of such reweighting is one of the issues we aim to improve in the future.

\subsection{Error Analysis}

The uncertainty analysis propagates the experimental errors from the data to the PDFs. The Hessian method, used in EPS09, relies on a quadratic approximation of $\chi^{2}$ around its minimum $\chi_{0}^{2}$

$$
\chi^{2}\left(\left\{a_{i}\right\}\right) \approx \chi_{0}^{2}+\sum_{i j} \delta a_{i} H_{i j} \delta a_{j}=\chi_{0}^{2}+\sum_{i} z_{i}^{2},
$$

where the $\delta a_{i}$ s are the deviations of the original fit parameters $a_{i}$ from the best-fit values. The key point is that the Hessian matrix $H_{i j}$ can be diagonalized thereby finding an uncorrelated basis $\left\{z_{i}\right\}$. Relying to so called $90 \%$ confidence criterion, we find that that distorting the best fit in the directions of each $z_{i}$ such that the $\chi^{2}$ changes by 50 , still gives a resonable agreement with the data. These corners of parameters define the uncertainty sets $S_{i}^{ \pm}$facilitiating the estimation of the upper $\backslash$ lower error in any PDF-dependent quantity $\mathrm{X}$ with best-fit prediction $X\left(S_{0}\right)$, by

$$
\Delta X^{+\backslash-}=\sqrt{\sum_{i} \max \backslash \min \left[X\left(S_{i}^{+}\right)-X\left(S_{0}\right), X\left(S_{i}^{-}\right)-X\left(S_{0}\right), 0\right]^{2}} .
$$

\subsection{Results}

We summarize the resulting nuclear effects in PDFs by Figure 1 which shows the nuclear effects in Lead at a low scale $Q^{2}=1.69 \mathrm{GeV}^{2}$ and also at a higher scale $Q^{2}=100 \mathrm{GeV}^{2}$ to demonstrate the effect of DGLAP evolution. For lighter nuclei the effects are closer to unity. The blue bands denote the EPS09-uncertainty. We also show the results from two alternative analyses $[12,13]$ helping to appreciate the well and badly constrained components of the nuclear effects.

\section{Recent Activities}

For studying the universality of nPDFs further, data from other processes are always welcome. One of such processes is the neutrino beam induced DIS. It has been suggested [9] that the NuTeV $v$ Fe-data [10] favors different nuclear effects than extracted from charged lepton DIS and DY processes. However, our analysis [11] with a larger set of data implies that this is a sheer artefact brought about looking only at the $\mathrm{NuTeV}$ data that appear to contain internal incosistencies.

In order to cleanly probe the nPDFs, an electron-ion collider like the planned LHeC would be an ideal machine. By fitting to a simulated $\mathrm{LHeC}$ data, we have found that such data would dramatically improve the determination of small- $x$ gluons and sea quarks. Another promising probe for nPDFs, that could be available before the realization of an electron-ion collider, would be the proton-ion runs at LHC. We have found that e.g. production of heavy electroweak bosons in such a collisions would provide a surprisingly neat tool to investigate the nuclear modifications to the PDFs with not much sensitivity to the underlying set of free proton PDFs. Both results mentioned here will be published shortly. 


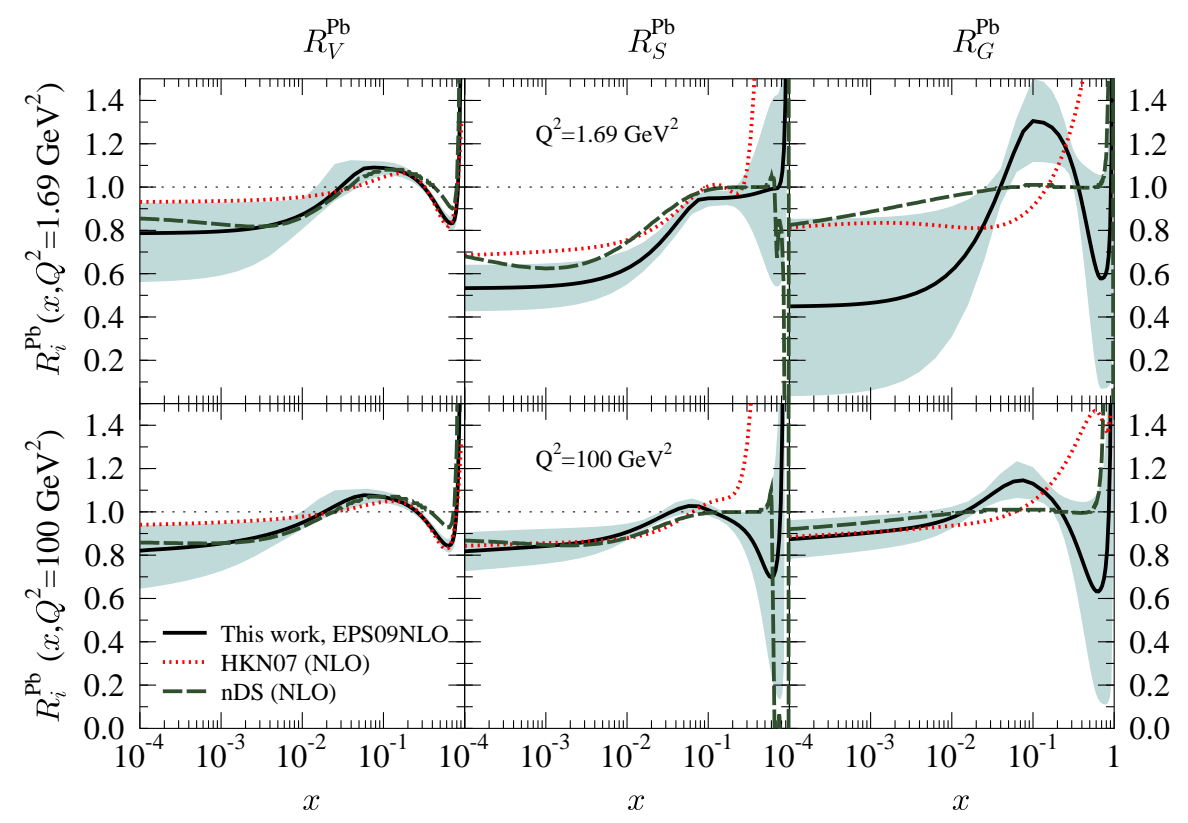

Figure 1: The nuclear effects for valence quarks $R_{V}$, sea quarks $R_{S}$, and gluons $R_{G}$ in Lead. The NLO results from EPS09, HKN07 [12], and nDS [13] are shown at two values of $Q^{2}$.

\section{References}

[1] K. J. Eskola, V. J. Kolhinen and P. V. Ruuskanen, Nucl. Phys. B 535 (1998) 351 [arXiv:hep-ph/9802350].

[2] K. J. Eskola, V. J. Kolhinen and C. A. Salgado, Eur. Phys. J. C 9 (1999) 61 [arXiv:hep-ph/9807297].

[3] K. J. Eskola, V. J. Kolhinen, H. Paukkunen and C. A. Salgado, JHEP 0705 (2007) 002 [arXiv:hep-ph/0703104].

[4] K. J. Eskola, H. Paukkunen and C. A. Salgado, JHEP 0807 (2008) 102 [arXiv:0802.0139 [hep-ph]].

[5] K. J. Eskola, H. Paukkunen and C. A. Salgado, JHEP 0904 (2009) 065 [arXiv:0902.4154 [hep-ph]].

[6] R. Sassot, M. Stratmann and P. Zurita, Phys. Rev. D 81 (2010) 054001 [arXiv:0912.1311 [hep-ph]].

[7] D. Stump, J. Huston, J. Pumplin, W. K. Tung, H. L. Lai, S. Kuhlmann and J. F. Owens, JHEP 0310 (2003) 046 [arXiv:hep-ph/0303013].

[8] H. L. Lai, M. Guzzi, J. Huston, Z. Li, P. M. Nadolsky, J. Pumplin and C. P. Yuan, arXiv:1007.2241 [hep-ph].

[9] I. Schienbein, J. Y. Yu, C. Keppel, J. G. Morfin, F. Olness and J. F. Owens, Phys. Rev. D 77 (2008) 054013 [arXiv:0710.4897 [hep-ph]].

[10] M. Tzanov et al. [NuTeV Collaboration], Phys. Rev. D 74 (2006) 012008 [arXiv:hep-ex/0509010].

[11] H. Paukkunen and C. A. Salgado, JHEP 1007 (2010) 032 [arXiv:1004.3140 [hep-ph]].

[12] M. Hirai, S. Kumano and T. H. Nagai, Phys. Rev. C 76 (2007) 065207 [arXiv:0709.3038 [hep-ph]].

[13] D. de Florian and R. Sassot, Phys. Rev. D 69 (2004) 074028 [arXiv:hep-ph/0311227]. 